\title{
INTERPRETATION OF STRONG SHORT-TERM CENTRAL PERTURBATIONS IN THE LIGHT CURVES OF MODERATE-MAGNIFICATION MICROLENSING EVENTS
}

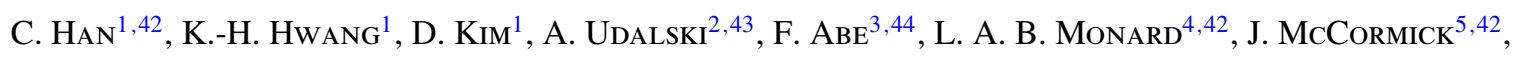 \\ AND
}

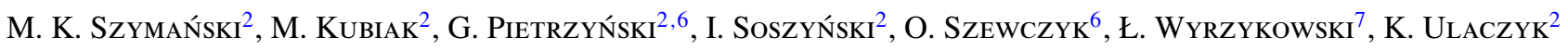

(The OGLE Collaboration),

I. A. Bond ${ }^{8}$, C. S. Botzler ${ }^{9}$, A. Fukui ${ }^{3}$, K. Furusawa ${ }^{3}$, J. B. Hearnshaw ${ }^{10}$, Y. Itow ${ }^{3}$, K. KamiYa ${ }^{3}$, P. M. Kilmartin ${ }^{11}$,

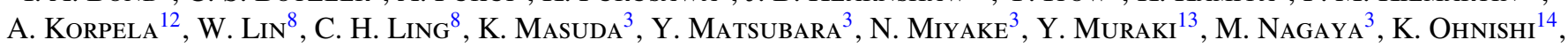
T. Tokumura ${ }^{3}$, Y. C. Perrotr ${ }^{9}$, N. Rattenbury ${ }^{15}$, To. Saito ${ }^{16}$, T. Sako ${ }^{3}$, L. Skuljan ${ }^{8}$, D. S. Sullivan ${ }^{12}$, T. Sumi $^{3}$, W. L. Sweatman ${ }^{8}$, P. J. Tristram ${ }^{11}$, P. C. M. Yock ${ }^{9}$

(The MOA COLlaboration),

\section{W. Allen ${ }^{17}$, G. W. Christie ${ }^{18}$, D. L. DePoy ${ }^{19}$, S. Dong ${ }^{20}$, B. S. Gaudi ${ }^{20}$, A. Gould ${ }^{20}$, C. $_{\text {U. }}$ LeE $^{21}$, T. NAtusCh $^{22}$,} B.-G. PARK $^{21}$, R. W. PogGE ${ }^{20}$

(The $\mu$ FUN COLlaboration),

M. D. Albrow ${ }^{10}$, A. Allan ${ }^{23}$, V. Batista ${ }^{24}$, J. P. Beaulieu ${ }^{24}$, D. P. BennetT ${ }^{25}$, S. Brillant ${ }^{26}$, M. Bode $^{27}$, D. M. Bramich ${ }^{28}$, M. Burgdorf ${ }^{27}$, J. A. R. Caldwell ${ }^{29}$, H. Calitz ${ }^{30}$, A. Cassan ${ }^{31}$, E. Corrales $^{24}$, S. Dieters ${ }^{24,32}$, D. D. Prester ${ }^{33}$,

M. Dominik ${ }^{34}$, J. Donatowicz ${ }^{35}$, P. Fouque ${ }^{36}$, J. Greenhill ${ }^{32}$, K. Hill ${ }^{32}$, M. Hoffman ${ }^{30}$, K. Horne ${ }^{34}$, U. G. Jørgensen ${ }^{37}$, N. Kains ${ }^{34}$, D. Kubas ${ }^{26}$, J. B. Marquette ${ }^{24}$, R. Martin ${ }^{38}$, P. Meintjes ${ }^{30}$, J. Menzies ${ }^{39}$, K. R. Pollard ${ }^{10}$,

K. C. $\mathrm{SAhu}^{40}$, C. Snodgrass ${ }^{26}$, I. Steele ${ }^{27}$, R. Street ${ }^{41}$, Y. Tsapras ${ }^{41}$, J. Wambsganss ${ }^{36}$, A. Williams ${ }^{38}$, and M. Zub ${ }^{31}$

(The PLANET/RoboNet Collaboration)

${ }^{1}$ Department of Physics, Chungbuk National University, Cheongju 361-763, Republic of Korea

${ }^{2}$ Warsaw University Observatory, Al. Ujazdowskie 4, 00-478 Warszawa, Poland

${ }^{3}$ Solar-Terrestrial Environment Laboratory, Nagoya University, Nagoya, 464-8601, Japan

${ }^{4}$ Bronberg Observatory, Centre for Backyard Astrophysics Pretoria, South Africa

${ }^{5}$ Farm Cove Observatory, Centre for Backyard Astrophysics, Pakuranga, Auckland New Zealand

${ }^{6}$ Universidad de Concepción, Departamento de Fisica, Casilla 160-C, Concepción, Chile

${ }^{7}$ Institute of Astronomy Cambridge University, Madingley Road, CB3 OHA Cambridge, UK

${ }^{8}$ Institute of Information and Mathematical Sciences, Massey University, Private Bag 102-904, North Shore Mail Centre, Auckland, New Zealand

${ }^{9}$ Department of Physics, University of Auckland, Private Bag 92019, Auckland, New Zealand

${ }^{10}$ University of Canterbury, Department of Physics and Astronomy, Private Bag 4800, Christchurch 8020, New Zealand

${ }^{11}$ Mount John Observatory, P.O. Box 56, Lake Tekapo 8770, New Zealand

${ }^{12}$ School of Chemical and Physical Sciences, Victoria University, Wellington, New Zealand

${ }^{13}$ Department of Physics, Konan University, Nishiokamoto 8-9-1, Kobe 658-8501, Japan

${ }^{14}$ Nagano National College of Technology, Nagano 381-8550, Japan

15 Jodrell Bank Centre for Astrophysics, University of Manchester, Manchester, M13 9PL, UK

${ }^{16}$ Tokyo Metropolitan College of Industrial Technology, Tokyo 116-8523, Japan

${ }^{17}$ Vintage Lane Observatory, Blenheim, New Zealand

${ }_{18}$ Auckland Observatory, Auckland, New Zealand

${ }^{19}$ Department of Physics, Texas A\&M University, College Station, TX, USA

${ }^{20}$ Department of Astronomy, Ohio State University, 140 West 18 th Avenue, Columbus, OH 43210, USA

${ }^{21}$ Korea Astronomy and Space Science Institute, Daejeon 305-348, Republic of Korea ${ }_{22}$ AUT University, Auckland, New Zealand

${ }^{23}$ School of Physics, University of Exeter, Stocker Road, Exeter EX4 4QL, UK

${ }^{24}$ Institut d'Astrophysique de Paris, 98bis Boulevard Arago, 75014 Paris, France

${ }^{25}$ Department of Physics, Notre Dame University, Notre Dame, IN 46556, USA

${ }^{26}$ European Southern Observatory, Alonso de Cordova 3107, Casilla 19001, Vitacura, Santiago 19, Chile

${ }^{27}$ Astrophysics Research Institute, Liverpool John Moores University, Twelve Quays House, Egerton Wharf, Birkenhead CH41 1LD, UK

${ }^{28}$ Issac Newton Group, Apartado de Correos 321, E-38700 Santa Cruz de La Palma, Spain

${ }^{29}$ McDonald Observatory, 16120 St Hwy Spur 78, Fort Davis, TX 79734, USA

${ }^{30}$ Department of Physics, Boyden Observatory, University of the Free State, Bloemfontein 9300, South Africa

${ }^{31}$ Astronomisches Rechen-Institut, Zentrum für Astronomie, Heidelberg University, Mönchhofstr. 12-14, 69120 Heidelberg, Germany

${ }^{32}$ University of Tasmania, School of Maths and Physics, Private bag 37, GPO Hobart, Tasmania 7001, Australia

${ }^{33}$ Physics Department, Faculty of Arts and Sciences, University of Rijeka, 51000 Rijeka, Croatia

${ }^{34}$ SUPA, Physics and Astronomy, North Haugh, St Andrews, KY16 9SS, UK

${ }^{35}$ Technical University of Vienna, Department of Computing, Wiedner Hauptstrasse 10, Vienna, Austria

${ }^{36}$ LATT, Universite de Toulouse, CNRS, 14, avenue Edouard Belin, 31400 Toulouse, France

${ }^{37}$ Niels Bohr Institute, Astronomical Observatory, Juliane Maries Vej 30, DK-2100 Copenhagen, Denmark

${ }^{38}$ Perth Observatory, Walnut Road, Bickley, Perth 6076, Australia

${ }^{39}$ South African Astronomical Observatory, P.O. Box 9 Observatory 7935, South Africa

${ }^{40}$ Space Telescope Science Institute, 3700 San Martin Drive, Baltimore, MD 21218, USA

${ }^{41}$ Las Cumbres Observatory Global Telescope Network, 6740B Cortona Dr, Suite 102, Goleta, CA, 93117, USA

Received 2009 June 11; accepted 2009 September 11; published 2009 October 19

\section{ABSTRACT}

To improve the planet detection efficiency, current planetary microlensing experiments are focused on highmagnification events searching for planetary signals near the peak of lensing light curves. However, it is known that 
central perturbations can also be produced by binary companions and thus it is important to distinguish planetary signals from those induced by binary companions. In this paper, we analyze the light curves of microlensing events OGLE-2007-BLG-137/MOA-2007-BLG-091, OGLE-2007-BLG-355/MOA-2007-BLG-278, and MOA2007-BLG-199/OGLE-2007-BLG-419, for all of which exhibit short-term perturbations near the peaks of the light curves. From detailed modeling of the light curves, we find that the perturbations of the events are caused by binary companions rather than planets. From a close examination of the light curves combined with the underlying physical geometry of the lens system obtained from modeling, we find that the short timescale caustic-crossing feature occurring at a low or a moderate base magnification with an additional secondary perturbation is a typical feature of binary-lens events and thus can be used for the discrimination between the binary and planetary interpretations.

Key words: gravitational lensing - planetary systems

Online-only material: color figures

\section{INTRODUCTION}

The microlensing signal of a planet is characterized by a shortterm perturbation to the smooth standard single-lens light curve of the primary-induced lensing event occurring on a background source star (Mao \& Paczyński 1991; Gould \& Loeb 1992). The duration of the perturbation is several days for a gas giant and several hours for an Earth-mass planet, while the typical cadence of lensing surveys is roughly a day. As a result, it is difficult to detect planets from the survey observations alone. To achieve the cadence required to detect short-term planetary signals, current planetary lensing searches are being conducted by combining survey and follow-up observations, in which the survey observations (OGLE: Udalski et al. 2003; MOA: Bond et al. 2002) aim to maximize the number of detections of lensing events by monitoring a large area of sky, and follow-up observations (PLANET: Albrow et al. 1998; Micro-FUN: Ghosh et al. 2004) are focused on the intensive monitoring of the events detected by the survey observations. However, the number of telescopes available for follow-up observations is far smaller than would be needed to monitor all the detected events from the survey observations. As a result, follow-up observations are selectively conducted for events that can maximize the planet detection probability. Currently, the highest priority is given to high-magnification events. For these events, the planet detection efficiency is high because the source trajectory always passes close to the region of central perturbations. In addition, the perturbation occurs near the peak of the light curve and thus follow-up observations can be prepared in advance.

Although the strategy to detect central perturbations has an important advantage of high sensitivity to planets, it also has a shortcoming of difficulty in the interpretation of observed signals. One important cause of this difficulty is that the perturbation near the peak of a lensing light curve can be produced not only by a planet but also by a wide or a close binary companion with a mass roughly equal to that of the primary. Fortunately, the perturbation patterns produced by the planetary and the binary companion are intrinsically different (Han \& Gaudi 2008; Han 2009a), and thus it is in principle possible to discriminate between the planetary and binary interpretations. However, this discrimination usually requires detailed modeling of the light curve, which demands a time-consuming search for a solution of the lensing parameters in the vast space of many parameters. Therefore, simple diagnostics that can be used for the discrimination between the two interpretations will be

\footnotetext{
${ }^{42}$ Microlensing Follow Up Network ( $\mu$ FUN).

${ }^{43}$ Optical Gravitational Lens Experiment (OGLE).

${ }^{44}$ Microlensing Observations in Astrophysics (MOA) Collaboration.
}

useful not only for the immediate identification of the nature of the perturbation but also for the preparation of observational strategies for better characterization of planetary systems.

In this paper, we present the results of the analysis of three binary-lens events with very strong short-term signals near the peaks of the light curves. From the investigation of the light curves combined with the underlying physical geometry of the lens system, we find and present several features in lensing light curves that can be used as diagnostic tools for the discrimination between the planetary and binary interpretations of observed events.

The paper is organized as follows. In Section 2, we briefly describe the general features of central perturbations produced by a planet and a binary companion. In Section 3, we discuss the data used in our analysis. In Section 4, we explain the modeling procedure and present the best-fit lensing parameters of the individual lensing events obtained from the modeling. In Section 5, we present the features in the light curves that characterize the binary nature of the events and discuss the usability of the features in distinguishing the planetary and binary interpretations. We summarize the results and conclude in Section 6.

\section{PLANETARY AND BINARY CENTRAL CAUSTICS}

Lens systems composed of multiple masses can produce strong perturbations when the trajectory of a source star passes close to a caustic. The caustic represents the source star position at which the magnification of a point source becomes infinite. For lenses consisting of two masses, the set of caustics form closed curves each of which is composed of concave curves that meet at cusps.

Binary lenses can have one, two, or three sets of caustics depending on the separation between the lens components. If the two masses are separated by approximately an Einstein radius, then there is a single six-cusp caustic. If the separation is substantially closer than the Einstein radius (close binary), there is a single four-cusp central caustic and two small outlying three-cusp caustics. If the masses are separated by substantially more than the Einstein radius (wide binary), there are two fourcusp caustics, each of which is associated with a member of the binary. The short-term central perturbation of a binary-lens event can occur in two different ways. The first case is when the trajectory of a source star passes close to the central caustic of a close binary. The other case happens when the trajectory passes close to one of the two caustics of a wide-separation binary. For both wide and close binaries, the central caustics have a diamond shape although there is some variation depending on the separation and the mass ratio between the two binary components. 


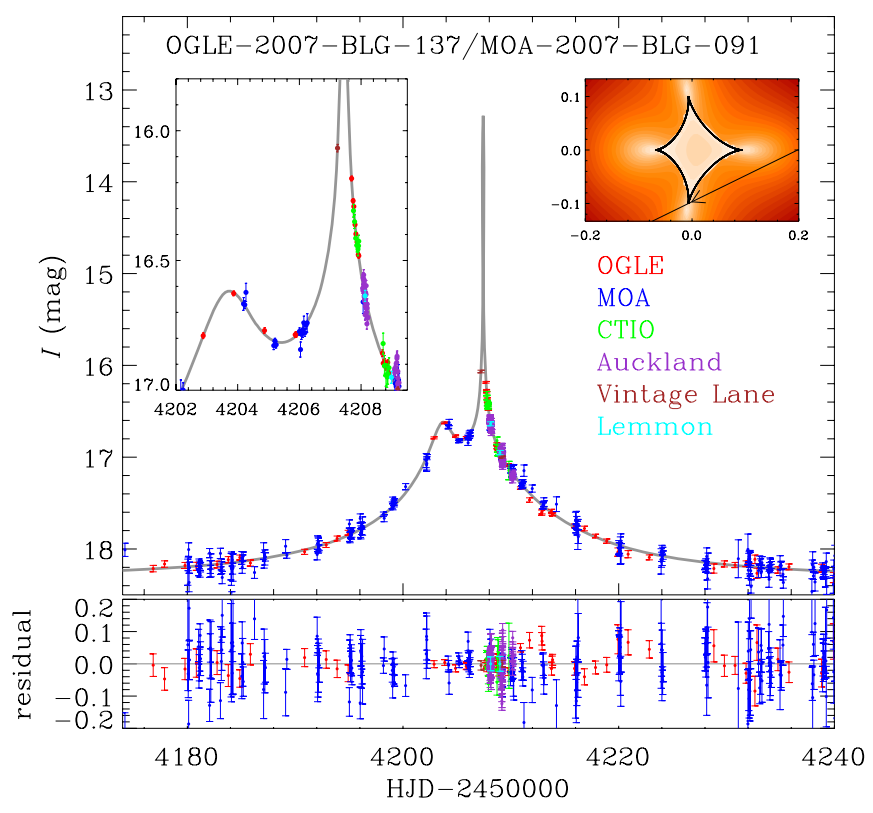

Figure 1. Data and best-fit model of the microlensing event OGLE-2007-BLG137/MOA-2007-BLG-091. The left inset shows a blowup of the region of major perturbation. The right inset shows the geometry of the source trajectory with respect to the caustic location. The coordinates are centered at the "center of magnification" of the lens system (see the definition in the text) and the abscissa and the ordinate are parallel with and normal to the binary axis, respectively. Heavier mass is on the left. The temperature scale represents the magnification where a brighter tone represents higher magnification. The bottom panel represents the residual from the model light curve.

(A color version of this figure is available in the online journal.)

For planetary lensing, the central caustic refers to a small fourcusp caustic located close to the primary lens. The size of the planetary central caustic as measured by the separation between the two cusps located on the primary-planet axis is related to the planetary lensing parameters by Chung et al. (2005)

$$
\Delta \xi_{\mathrm{c}} \sim \frac{4 q}{\left(s-s^{-1}\right)^{2}},
$$

where $s$ represents the projected primary-planet separation normalized by the Einstein radius corresponding to the total mass of the lens system, $\theta_{\mathrm{E}}$, and $q$ is the planet/primary mass ratio. While the size of the central caustic depends on both the mass ratio and the separation, its shape depends solely on the separation. When the shape is quantified as the ratio of the vertical width $\Delta \eta_{\mathrm{c}}$ to the horizontal width $\Delta \xi_{\mathrm{c}}$, the width ratio is related to the planetary separation by (Chung et al. 2005)

$$
\frac{\Delta \eta_{\mathrm{c}}}{\Delta \xi_{\mathrm{c}}} \sim \frac{\left(s-s^{-1}\right)^{2}\left|\sin ^{3} \phi\right|}{\left(s+s^{-1}-2 \cos \phi\right)^{2}},
$$

where $\cos \phi=(3 / 4)\left(s+s^{-1}\right)\left\{1-\left[1-(32 / 9)\left(s+s^{-1}\right)^{-2}\right]^{1 / 2}\right\}$. For the range of the planetary separations within which the planet detection efficiency is not negligible, the width ratio of the planetary central caustic is substantially smaller than unity (Han 2009b), implying that the caustic is elongated along the primary-planet axis. The caustic has an arrowhead shape where three cusps are located close to the primary and the other cusp is located at the tip of the arrow pointing toward the direction of the planet. For a given mass ratio, a pair of central caustics with separations $s$ and $s^{-1}$ is identical to the first order of approximation (Dominik 1999; An 2005).

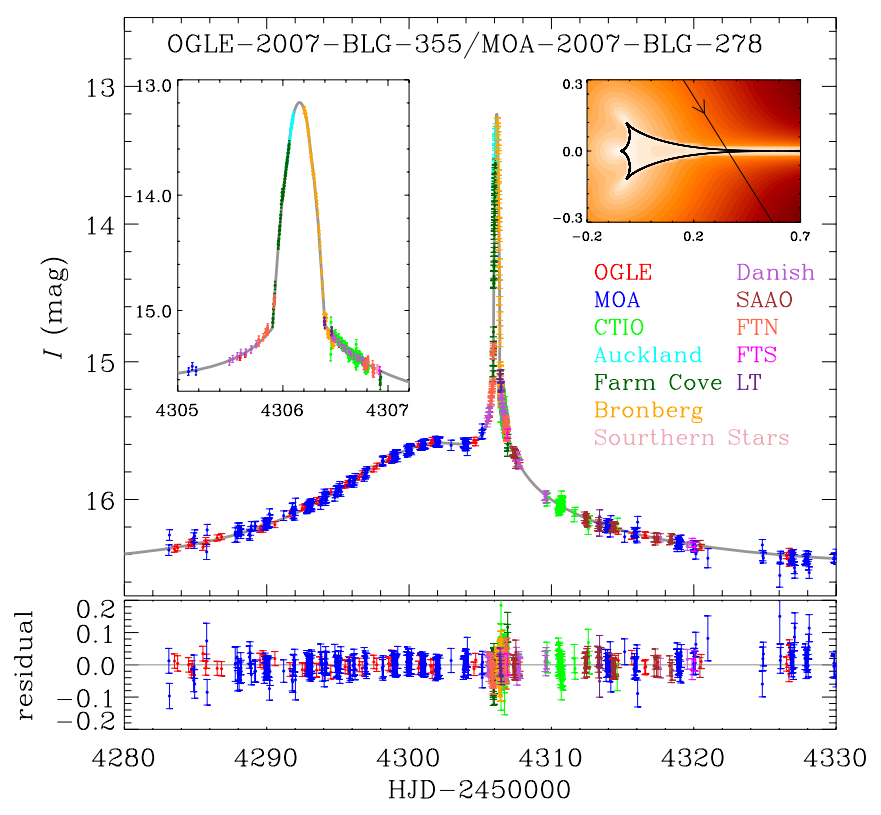

Figure 2. Data and best-fit model of OGLE-2007-BLG-355/MOA-2007-BLG278. Notations are the same as in Figure 1.

(A color version of this figure is available in the online journal.)

\section{OBSERVATIONS}

The events we analyze include OGLE-2007-BLG-137/ MOA-2007-BLG-091, OGLE-2007-BLG-355/MOA-2007BLG-278, and MOA-2007-BLG-199/OGLE-2007-BLG-419. All these events were observed in the 2007 microlensing observation season. The light curves of the individual events are presented in Figures 1-3. They show a common feature of strong perturbations occurring near the peaks of the light curves. Due to the location of the perturbations similar to those of central perturbations induced by planets, all events were alerted by survey observations and intensively monitored by follow-up observations.

The lensing event OGLE-2007-BLG-137/MOA-2007-BLG091 was alerted on 2007 April 15 by the OGLE collaboration as a possible event with an anomaly near the peak, using the $1.3 \mathrm{~m}$ Warsaw telescope of Las Campanas Observatory in Chile. In response to the alert, follow-up observations were conducted by the $\mu$ FUN team with various telescopes, including the $1.3 \mathrm{~m}$ of CTIO in Chile, the $0.4 \mathrm{~m}$ of Auckland Observatory, the $0.4 \mathrm{~m}$ of Vintage Lane Observatory in New Zealand, and the $1.0 \mathrm{~m}$ of Mt. Lemmon Observatory in Arizona, United States. The MOA collaboration independently discovered the event by using the $1.8 \mathrm{~m}$ of Mt. John in New Zealand. From these observations, a total of 457, 1119, 14, 65, 2, and 2 images were obtained from Las Campanas, Mt. John, CTIO, Auckland, Vintage Lane, and Mt. Lemmon, respectively.

The event OGLE-2007-BLG-355/MOA-2007-BLG-278 was detected independently by the OGLE and MOA survey groups and its anomalous behavior was noticed by the SIGNALMEN, a software developed by Dominik (2007) for early detection of anomalies and now integrated part of the ARTEMiS system (Dominik 2008), on 2007 July 20. Soon after, the $\mu$ FUN and PLANET/RoboNet teams conducted intensive follow-up observations by using the $1.3 \mathrm{~m}$ of CTIO, the $0.4 \mathrm{~m}$ of Auckland Observatory, the $0.36 \mathrm{~m}$ of Farm Cove Observatory in New Zealand, the $0.28 \mathrm{~m}$ of Southern Stars Observatory in Tahiti, the $0.36 \mathrm{~m}$ of Bronberg Observatory in South Africa, $1.54 \mathrm{~m}$ 


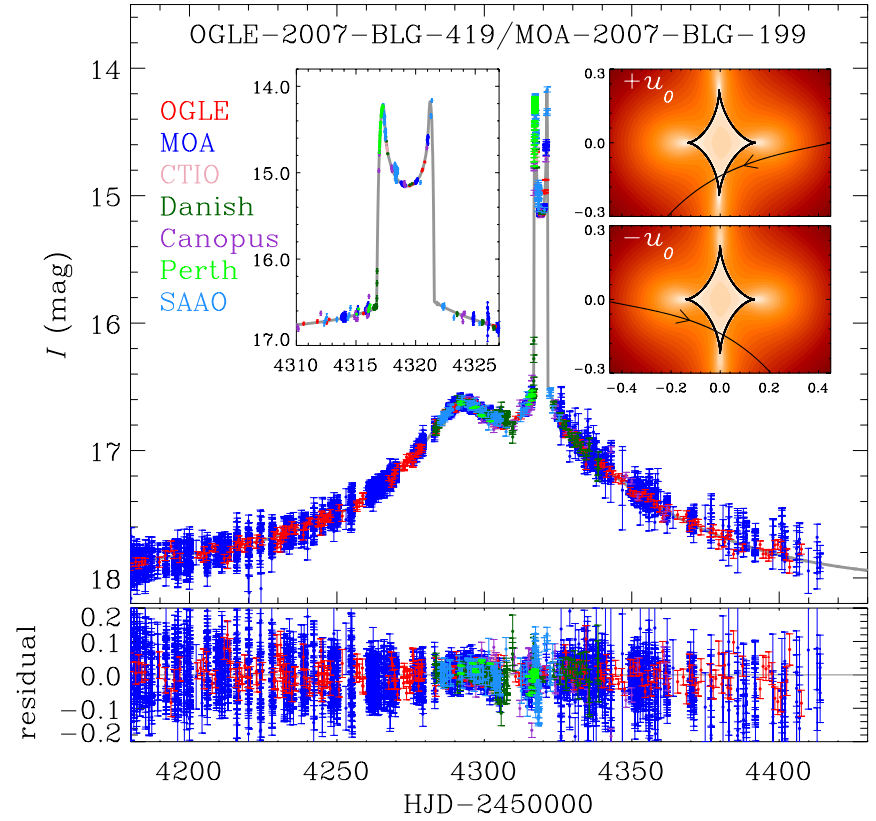

Figure 3. Data and best-fit model of MOA-2007-BLG-199/OGLE-2007-BLG419. Notations are the same as in Figure 1 except that there are two insets of source trajectory geometry for the two degenerate models. The presented model light curve is for " $+u_{0}$ " solution (see Table 1), which has smaller $\Delta \chi^{2}$ by $\sim 90$. (A color version of this figure is available in the online journal.)

Danish Telescope of La Silla Observatory in Chile, the $1.0 \mathrm{~m}$ of SAAO in South Africa, $2.0 \mathrm{~m}$ Faulkes S. (FTS) Telescope in Australia, $2.0 \mathrm{~m}$ Faulkes N. (FTN) Telescope in Hawaii, and $2.0 \mathrm{~m}$ Liverpool Telescope (LT) in La Palma, Spain. Due to the prompt response to the alert, the perturbation was very densely resolved by the follow-up observations despite the fact that the perturbation lasted only about 1 day. The data consist of 591, 1325, 187, 21, 39, 3, 43, 40, 34, 3, 27, and 7 images obtained from Las Campanas, Mt. John, CTIO, Auckland, Farm Cove, Southern Star, Bronberg, La Silla, SAAO, FTS, FTN, and LT, respectively.

The event MOA-2007-BLG-199/OGLE-2007-BLG-419 is a long timescale event. The PLANET/RoboNet team first announced the anomalous behavior of the event on 2007 July 28 and conducted follow-up observations by using the $1.54 \mathrm{~m}$ Danish Telescope, the $1.0 \mathrm{~m}$ of Canopus Observatory, the $0.6 \mathrm{~m}$ of Perth Observatory in Australia, and the $1.0 \mathrm{~m}$ of SAAO. The $\mu$ FUN collaboration also observed the event by using the $1.3 \mathrm{~m}$ of CTIO. The event shows a clear signature of caustic crossing during HJD 2454316-2454322. The data consist of 1233, 4108, 126, 56, 52, 130, and 15 images obtained from Las Campanas, Mt. John University, La Silla, Canopus, Perth, SAAO, and CTIO, respectively.

For the analysis of the light curves, we use the photometry results that were reduced by the individual groups using their own photometry codes.

\section{MODELING}

Binary-lens modeling requires inclusion of various parameters. The first set of three parameters is related to the geometry of a single-lens event. These include the Einstein timescale, $t_{\mathrm{E}}$, the time of maximum magnification, $t_{0}$, and the lens-source separation normalized by the Einstein radius $\theta_{\mathrm{E}}$ at the time of maximum magnification, $u_{0}$. For the description of the perturbation, an additional set of binary parameters is required. These parameters include the mass ratio $q$ and separation $s$ between the lens components and the angle of the source trajectory with respect to the binary axis, $\alpha$. In addition, the normalized source radius, $\rho_{\star} \equiv \theta_{\star} / \theta_{\mathrm{E}}$, is needed to describe the lensing magnification whenever the angular radius of the source star $\theta_{\star}$ plays an important role, such as the moment of a caustic crossing. For some events with long timescales, it is required to include the parallax parameters $\pi_{\mathrm{E}, N}$ and $\pi_{\mathrm{E}, E}$ to account for the deviation of the source motion from rectilinear motion caused by the parallactic motion of the Earth around the Sun (Gould 2000). The microlens parallax is defined by $\pi_{\mathrm{E}}=\mathrm{AU} / \tilde{r}_{\mathrm{E}}$, i.e., the ratio of the radius of the Earth's orbit around the Sun to the Einstein radius projected on the observer plane, $\tilde{r}_{\mathrm{E}}$. The subscripts " $N$ " and " $E$ " refer to the components of the parallax vector projected on the sky in the north and east celestial coordinates.

To determine the model parameters, we conduct a massive search for solutions over a broad range of mass ratios and separations. For this, we hold the binary parameters $s, q$, and $\alpha$ fixed at a grid of values, while the remaining parameters $\left(t_{\mathrm{E}}\right.$, $u_{0}, t_{0}$, and $\rho_{\star}$ ) are allowed to vary so that the model light curve results in minimum $\chi^{2}$ at each grid point. We use a Markov Chain Monte Carlo method for the $\chi^{2}$ minimization. Once the $\chi^{2}$ minima of the individual grid points are determined, the bestfit model is obtained by comparing the $\chi^{2}$ values. Although a brute search in the space of the binary parameters requires a large computation time, it is needed to investigate the existence of possible degenerate solutions.

For the computation of lensing magnification including the finite-source effect, we use the "magnification map" technique (Dong et al. 2006, 2009). In this technique, a magnification map is constructed for a given set of $s$ and $q$ by using the rayshooting method. Based on this map, the lensing magnification corresponding to a location of a source star on the map is computed by comparing the number density of rays within the source radius with the density on the lens plane. On the basis of this scheme, the computation is accelerated in several ways. First, the algorithm is designed to save computation time by limiting the range of ray shooting on the image (lens) plane to a narrow annulus encircling the Einstein ring of the primary lens. This is based on the fact that only the rays from this region arrive at the central perturbation region on the source plane. Second, the algorithm keeps the information of the positions of all the rays arriving at the target in the buffer memory space of a computer so that the information can be promptly used for fast computation of the magnification. Third, the finitesource magnification calculation based on ray-shooting is used only in the region near the caustic and a simple hexadecapole approximation (Pejcha \& Heyrovský 2009; Gould 2008) is used in other part of light curves.

In Figures 1-3, we present the model light curves superposed on the data of the individual events. The geometry of the source trajectory with respect to the caustic location for each event is presented in the inset of each figure. The coordinates of the inset are centered at the center of magnification around which the single-lens magnification best describes that of the binary lensing. The abscissa and ordinate are parallel with and normal to the binary axis, respectively. In Table 1, we also present the values of the individual lensing parameters. ${ }^{45}$ We note

\footnotetext{
45 For the event MOA-2007-BLG-199/OGLE-2007-BLG-419, we present two sets of solutions resulting from the known " $+u_{0} \leftrightarrow-u_{0}$ " degeneracy in parallax determination (Smith et al. 2003). We find that there exists no "jerk-parallax" degeneracy, which is another known degeneracy in parallax determination (Gould 2004; Park et al. 2004).
} 
Table 1

Best-fit Parameters

\begin{tabular}{|c|c|c|c|c|c|c|c|c|c|}
\hline Event (Model) & $s$ & $q$ & $\begin{array}{c}\alpha \\
(\operatorname{deg})\end{array}$ & $\begin{array}{c}t_{0} \\
\text { (HJD') }\end{array}$ & $u_{0}$ & $\begin{array}{c}t_{\mathrm{E}} \\
\text { (days) }\end{array}$ & $\rho_{\star}$ & $\pi_{\mathrm{E}, N}$ & $\pi_{\mathrm{E}, E}$ \\
\hline OGLE-2007-BLG-137/MOA-2007-BLG-091 & $0.43 \pm 0.05$ & $0.59 \pm 0.02$ & 153.95 & 4206.368 & 0.087 & 21.41 & 0.00054 & & \\
\hline OGLE-2007-BLG-355/MOA-2007-BLG-278 & $1.84 \pm 0.01$ & $0.24 \pm 0.01$ & 57.93 & 4303.072 & 0.334 & 14.73 & 0.00988 & & \\
\hline MOA-2007-BLG-199/OGLE-2007-BLG-419 $\left(+u_{0}\right)$ & $0.57 \pm 0.01$ & $0.88 \pm 0.01$ & 157.17 & 4313.460 & 0.122 & 116.31 & 0.00198 & 0.395 & 0.014 \\
\hline MOA-2007-BLG-199/OGLE-2007-BLG-419 $\left(-u_{0}\right)$ & $0.57 \pm 0.01$ & $1.00 \pm 0.01$ & 23.84 & 4312.963 & -0.125 & 119.44 & 0.00194 & -0.461 & 0.116 \\
\hline
\end{tabular}

Notes. HJD' $=$ HJD - 2450000. For the event OGLE-2007-BLG-419/MOA-2007-BLG-199, we present two sets of solutions resulting from the "+ $u_{0} \leftrightarrow-u_{0}$ " degeneracy in parallax determination. The " $+u_{0}$ " model provides a slightly better fit with a $\Delta \chi^{2}$ per degree of freedom of $\Delta \chi^{2} /$ dof $\simeq 90 / 5711$.

that modeling the light curve of the event MOA-2007-BLG199/OGLE-2007-BLG-419 with the basic binary parameters does not yield a satisfactory model and requires inclusion of additional parallax parameters. As a result, the trajectory of the event is curved. It is found that the determined mass ratios of the three lenses range from 0.24 to 1.0 , implying that the perturbations are produced by binary companions not planets. It is also found that the short strong perturbations are caused by crossing the tip of the caustic.

We note that including the parameters of higher-order effect, $\rho_{\star}$ and $\boldsymbol{\pi}_{\mathrm{E}}$, is necessary to obtain a good fit to the data. But the data reductions, which come directly from the pipelines, might not be adequate to measure these subtle parameters accurately. Since these parameters are irrelevant to the main scientific purpose of the paper, and since refined data reductions are typically very time-consuming, we present these higher-order parameters only for completeness.

\section{BINARY FEATURES}

The light curves of the events we analyzed are mainly characterized by the perturbation near the peak similar to the central perturbation caused by a planet. However, a close examination of the perturbations reveals several features that convey the binary nature of the perturbation. In this section, we discuss these features.

The first feature that gives a clue as to the nature of the events is the strength of the main perturbation. For all three events, the deviations from the smooth base of the light curve exceed 3 magnitudes. This strength suggests that the perturbations are caused by caustic crossings.

The second feature is the duration of the perturbation. For all events, the duration of the main perturbation is very short compared with their timescales. The short duration of the perturbation can be explained either by the caustic being tiny or by the source trajectory crossing the tip of a cuspy part of a caustic with a nonnegligible size.

The third feature is the base magnification just before and after the perturbation. The base magnification of a causticcrossing event is important for the characterization of the lens system because it gives a strong constraint on the size of the caustic. For the event OGLE-2007-BLG-355/MOA-2007BLG-278, the base magnification is only $A \sim 2$ (corresponding to the normalized lens-source separation of $u \sim 0.5$ ). This implies that the caustic size is half as large as the Einstein radius and thus the binary origin of the perturbation can be immediately noticed. The events OGLE-2007-BLG-137/ MOA-2007-BLG-091 and MOA-2007-BLG-199/OGLE-2007BLG-419 have moderate base magnifications of $A \sim 10$ (corresponding to $u \sim 0.1$ ). Considering that the size of the central caustic produced by a giant planet with a mass ratio $q \sim 10^{-3}$ located around the Einstein ring of the primary with
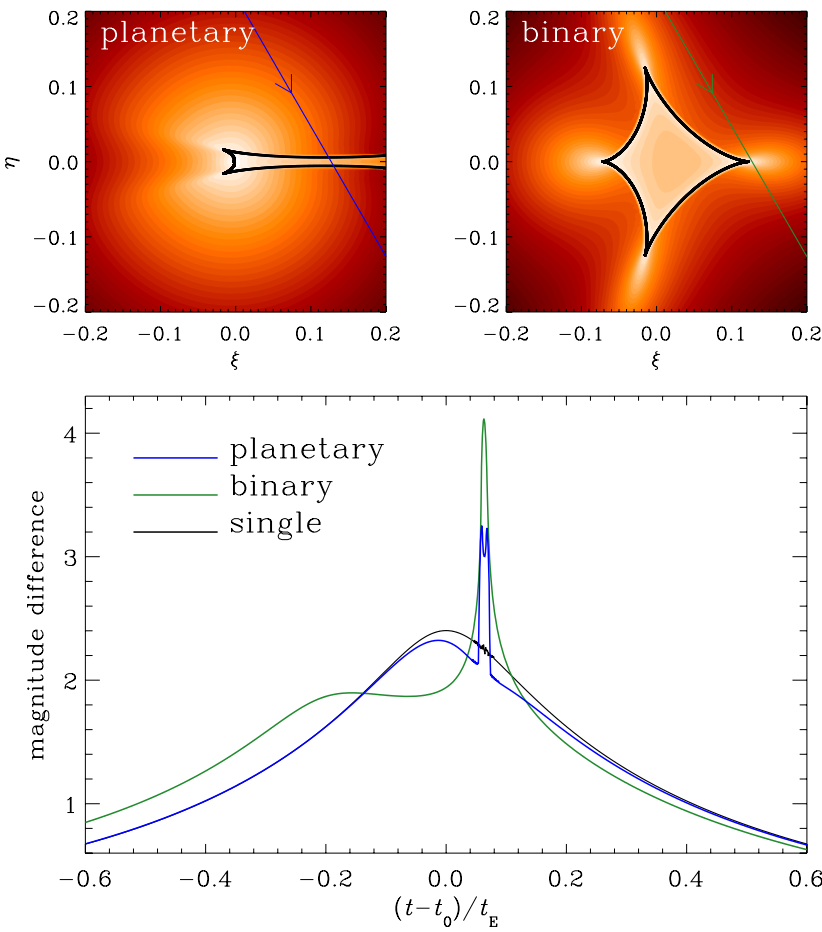

Figure 4. Magnification maps of a resonant planetary system (upper left panel) and a close binary system (upper right panel) in the regions around the central caustics of the individual systems. The lower panel shows the light curves resulting from the source trajectories marked in the upper panels.

(A color version of this figure is available in the online journal.)

a separation $s \sim 1.3$ is $\Delta \xi_{\mathrm{c}} \sim 0.01$, the chance that the main perturbations of these events are caused by planets is not high. However, one still cannot rule out the possibility of a big central caustic produced by a planet located very close to the Einstein ring (resonant planet).

The last feature is the additional secondary perturbation. The central caustic of a resonant planet can become large and thus planetary perturbations can occur at a moderate base magnification. Figure 4 shows the magnification maps of a resonant planetary system in the region around the central caustic. To produce a planetary perturbation at a low magnification, the source trajectory should pass close to the cusp corresponding to the tip of the arrow-head shaped planetary central caustic such as the one shown in Figure 4. In this case, it is difficult to produce an additional secondary perturbation because all the other cusps are remotely located on the opposite side of the caustic close to the primary. On the other hand, the central caustic produced by a binary companion has a diamond shape and thus the geometric chance to produce a secondary perturbation by passing an adjacent cusp of the caustic is high as demonstrated in Figure 4. Therefore, the existence of secondary perturbation strongly advocates the binary interpretation of the events. 
In summary, central perturbations that are characterized by short timescale caustic-crossing features occurring at a low or a moderate base magnification with an additional secondary perturbation are caused by binary companions. Considering that three events were detected during a single observation season, events with such a perturbation feature are common and the feature can be used as a diagnostic for the discrimination between the binary and planetary interpretations of perturbations.

\section{CONCLUSION}

We analyzed the light curves of three microlensing events with short-term perturbations near the peaks of the light curves. From detailed modeling of the light curves, we found that the perturbations of the events are caused by binary companions rather than planets. From a close examination of the light curves combined with the underlying physical geometry of the lens system obtained from modeling, we found that the short timescale caustic-crossing feature occurring at a low or a moderate base magnification with an additional secondary perturbation is a typical feature of binary-lens events and thus can be used for the discrimination between the binary and planetary interpretations.

We acknowledge the following support: National Research Foundation of Korea 2009-0081561 (CH); NSF AST-0757888 (AG,SD); NASA NNG04GL51G (DD, AG, RP); Polish MNiSW N20303032/4275 (AU); HST-GO-11311 (KS); NSF AST-0708890, NASA NNX07AL71G (DPB); MEXT19015005, JSPS18749004 (TS); Marden Fund of NZ (IAB, JBH, DJS, SLS, PCMY); Foundation for Research
Science and Technology of NZ (IAB, LS); Korea Astronomy and Space Science Institute (B-GP, C-UL); Deutsche Forschungsgemeinschaft (CSB); PPARC, EU FP6 program "ANGLES" (LW, NJR); PPARC (RoboNet); Dill Faulkes Educational Trust (Faulkes Telescope North); Grants JSPS18253002, JSPS20340052, and JSPS19340058 (MOA); Marsden Fund of NZ (IAB, PCMY); Foundation for Research Science and Technology of NZ. The operation of Mt. Canopus Observatory is supported in part by the financial contribution from David Warren.

\section{REFERENCES}

Albrow, M., et al. 1998, ApJ, 509, 687

An, J. H. 2005, MNRAS, 356, 1409

Bond, I. A., et al. 2002, MNRAS, 331, L19

Chung, S.-J., et al. 2005, ApJ, 630, 535

Dominik, M. 1999, A\&A, 329, 248

Dominik, M., et al. 2007, MNRAS, 380, 792

Dominik, M., et al. 2008, Astron. Nach., 329, 248

Dong, S., et al. 2006, ApJ, 642, 842

Dong, S., et al. 2009, ApJ, 698, 1826

Ghosh, H., et al. 2004, ApJ, 615, 450

Gould, A. 2000, ApJ, 542, 785

Gould, A. 2004, ApJ, 606, 319

Gould, A. 2008, ApJ, 681, 1593

Gould, A., \& Loeb, A. 1992, ApJ, 396, 104

Han, C. 2009a, ApJ, 691, L9

Han, C. 2009b, ApJ, 691, 452

Han, C., \& Gaudi, B. S. 2008, ApJ, 689, 53

Mao, S., \& Paczyński, B. 1991, ApJ, 374, L37

Park, B.-G., et al. 2004, ApJ, 609, 166

Pejcha, O., \& Heyrovský, B. 2009, ApJ, 690, 1772

Smith, M. C., Mao, S., \& Paczyński, B. 2003, MNRAS, 339, 925

Udalski, A., et al. 2003, Acta Astron., 53, 291

Yoo, J., et al. 2004, ApJ, 603, 139 